Andreas Fiolitakis, Peter R. Ess, Peter Gerlinger, Manfred Aigner, Modeling of heat transfer and differential diffusion in transported PDF methods, Combustion and Flame 161 (2014) 2107 - 2119

The original publication is available at www.elsevier.com

http://dx.doi.org/10.1016/j.combustflame.2014.01.021 


\title{
Modeling of heat transfer and differential diffusion in transported PDF methods
}

\author{
A. Fiolitakis ${ }^{\mathrm{a}}$, P. R. Ess ${ }^{\mathrm{a}}$, P. Gerlinger ${ }^{\mathrm{b}}$, M. Aigner ${ }^{\mathrm{a}}$ \\ ${ }^{a}$ German Aerospace Center (DLR), Pfaffenwaldring 38-40, 70569 Stuttgart, Germany \\ ${ }^{b}$ Institute of Combustion Technology of Aerospace Engineering, University of Stuttgart, \\ Pfaffenwaldring 38-40, 70569 Stuttgart, Germany
}

\begin{abstract}
In order to model the conditional diffusive heat and mass fluxes in the joint probability density function (PDF) transport equation of the thermochemical variables, the diffusive fluxes are decomposed into their Favre mean and fluctuation. While the mean flux appears to be closed, the contributions of fluctuating fluxes are modeled with the interaction by exchange with the mean (IEM) model. Usually, the contribution of the Favre averaged diffusive fluxes is neglected at high Reynolds numbers. Here, however, this term is included to account for molecular mixing in regions, where turbulent mixing is negligible. This model approach is applied in steady state Reynolds Averaged Navier-Stokes (RANS)/ transported PDF calculations to simulate the heat transfer of wall bounded flows as well as the stabilization of a hydrogenair flame at the burner tip. For both flow problems it is demonstrated that molecular transport is recovered in regions where turbulent mixing vanishes. In wall bounded flows this is the case in the viscous sublayer. Heat transfer studies show, that "mixing models" based on the high Reynolds number as-
\end{abstract}

Email address: andreas.fiolitakis@dlr.de (A. Fiolitakis) 
sumption fail to compute correctly the temperature field and the heat flux close to the wall. A similar situation occurs at the flame root of the investigated turbulent hydrogen-air jet flame, where turbulent mixing is still too weak to achieve a fast mixing of reactants. In this area differential diffusion effects are observed in the experiment, i.e. superequilibrium temperatures and nonlinear relations between the elemental mixture fractions of hydrogen and oxygen. It will be shown, that the presented model can successfully reproduce these effects, which underlines the necessity to include Favre averaged molecular diffusive fluxes in transported PDF methods.

Keywords:

Transported PDF, Differential Diffusion, Heat Transfer

\section{Introduction}

During the last decades the transported probability density function (PDF) method has received great attention for the calculation of turbulent, chemically reacting flows. The outstanding strength of this approach is that the effect of chemical reactions, i.e. the chemical source term, appears in closed form. One of the methods major drawbacks, however, are the unclosed conditional diffusive heat and mass fluxes. These terms, the mixing terms, account for the effect of mixing and are of paramount importance in order to predict flame extinction as well as flame stabilization. Since the introduction of the transported PDF method a large number of "mixing models" has been introduced in order to provide closure for these terms. Early examples are the coalescence and dispersion model (CD) of Curl [1] in 1963, the interaction by exchange with the mean (IEM) model of Villermaux and Dev- 
illon [2] in 1972, and the Linear-Mean-Square-Estimate (LMSE) of Dopazo and O'Brien [3] in 1974. Further improvements of the Curl model are introduced later by Janicka et al. [4] in 1979, Pope [5] in 1982, and Nooren et al. [6] in 1997. More recent examples include the Lagrangian-Fokker-Planck (LFP) model of Fox and Yeung [7], the Parameterized-Scalar-Profile (PSP) model of Meyer and Jenny [8, 9, 10, 11], the Mapping Closure (MP) of Pope [12] and Gao and O'Brien [13] (which is based on the work of Chen et al. [14]), and the Euclidean-Minimum-Spanning-Trees (EMST) of Subramaniam and Pope [15]. Usually, the assumption of high Reynolds numbers is invoked in these model approaches and the molecular transport is neglected [16]. However, there are situations in practice where the molecular transport of mass and heat is important. This concerns on one hand the heat transfer at walls. In the near wall region of a turbulent flow (in the viscous sublayer) viscosity dominates the transport of momentum. Consequently, molecular transport of heat becomes important in this region and must be included in "mixing models". Similar situations arise at the root of some turbulent, non-premixed flames which stabilize on the burner nozzle. In the near nozzle region the flow is essentially laminar and diffusive transport of species and heat is important for stabilizing the flame.

One way to treat molecular transport in transported PDF methods is introduced by Janicka et al. [4]. Based on the assumption of equal species diffusivities and assuming a Lewis number of one, the mixing terms are transformed, yielding a diffusive transport in physical space. Differential diffusion can not be treated by this method. Approaches on including heat conduction 
in transported PDF methods are discussed by Pozorski et al. [17]. Here a model for the inclusion of wall heat transfer in the one-point one-time PDF of temperature is presented. Since we are interested in the joint PDF of composition and enthalpy for the calculation of reacting flows, a different modeling approach is required. A method for treating molecular transport in hybrid Large-Eddy-Simulation (LES)/Filtered-Density-Function (FDF) techniques is discussed by McDermott et al. [18] in their work of 2007. In 2011 this method has been extended by Viswanathan et al. [19] to LES/PDF calculations. In both works a decomposition of the diffusive heat and mass fluxes into their mean and fluctuation is employed. This approach is utilized in the present work to derive a similar model for the application in hybrid ReynoldsAveraged-Navier-Stokes (RANS)/composition PDF calculations.

This methodology is briefly outlined in section 2 and 3. The capability of the method to predict heat transfer is discussed in section 4 where two test cases are investigated. The first test case concerns the heat transfer in a channel, where transported PDF calculations are compared to finite-volume method results. In the second test case the thermal entrance region of a fully developed turbulent pipe flow is calculated by means of the transported PDF method. Experimental data of Abbrecht and Churchill [20] are available for model validation. In section 5 the method is finally applied to the calculation of a non-premixed turbulent hydrogen-air flame [21, 22]. It is known that this flame is stabilized at the flame root by differential diffusion [22]. Experimental data are available to quantify the agreement between model predictions and measurements. 


\section{PDF Transport equation}

In order to compute expectations of the thermochemical state variables, a transport equation for the density weighted form of the PDF, the mass density function (MDF) $\mathscr{F}_{\phi}$, is solved. Assuming a low-Mach number flow and a thermally perfect gas [23] at constant pressure, the thermodynamic state is determined by the specific enthalpy $h$ and the composition in terms of mass fractions $Y_{i}$. For these variables the one-point, one-time MDF transport equation reads (Einstein notation) [24]

$$
\begin{aligned}
\frac{\partial \mathscr{F}_{\phi}}{\partial t}+\frac{\partial}{\partial x_{\alpha}}\left(\left\langle u_{\alpha} \mid \boldsymbol{\psi}\right\rangle \mathscr{F}_{\phi}\right) & =\frac{\partial}{\partial h}\left(\mathscr{F}_{\phi}\left\langle\frac{1}{\rho} \frac{\partial q_{\alpha}}{\partial x_{\alpha}} \mid \psi\right\rangle\right) \\
+ & \frac{\partial}{\partial Y_{i}}\left(\mathscr{F}_{\phi}\left\langle\frac{1}{\rho} \frac{\partial j_{i \alpha}}{\partial x_{\alpha}} \mid \psi\right\rangle\right)-\frac{\partial}{\partial Y_{i}}\left(\mathscr{F}_{\phi} \frac{\dot{m}_{i}}{\rho}\right) .
\end{aligned}
$$

In this equation $\rho$ denotes the density, $u_{\alpha}$ the convective flow velocity, $\dot{m}_{i}$ the chemical source term, and $j_{i \alpha}$ and $q_{\alpha}$ the diffusive heat and mass fluxes. The operator $\langle\bullet \mid \boldsymbol{\psi}\rangle$ denotes conditional expectations, where $\boldsymbol{\psi}$ is the sample space vector of the random vector $\phi=\left(h, Y_{i}\right)^{T}$. Terms involving conditional expectations require modeling. In order to model the conditional convective spatial transport $\left\langle u_{\alpha} \mid \psi\right\rangle$, the velocity is decomposed into its Favre mean $\tilde{u}_{\alpha}$ and fluctuation $u_{\alpha}^{\prime \prime}$. This yields $\left\langle u_{\alpha} \mid \boldsymbol{\psi}\right\rangle=\tilde{u}_{\alpha}+\left\langle u_{\alpha}^{\prime \prime} \mid \boldsymbol{\psi}\right\rangle$. Employing the gradient diffusion model of Pope [25] to close the contribution of the conditional expectation of fluctuating velocity to the spatial transport of the MDF yields

$$
\left\langle u_{\alpha} \mid \boldsymbol{\psi}\right\rangle \mathscr{F}_{\phi}=\tilde{u}_{\alpha} \mathscr{F}_{\phi}-\langle\rho\rangle D_{T} \frac{\partial}{\partial x_{\alpha}}\left(\frac{\mathscr{F}_{\phi}}{\langle\rho\rangle}\right),
$$

where $D_{T}$ denotes the turbulent diffusivity and the operator $\langle\bullet\rangle$ expectations. The above method of decomposing a variable into its mean and fluctuation 
is used by McDermott et al. [18] in order to model the conditional diffusive fluxes of heat and mass. The diffusive heat flux is given in this work by a simplified Fickian approach

$$
j_{i \alpha}=-\rho D_{i} \frac{\partial Y_{i}}{\partial x_{\alpha}}+Y_{i} \sum_{j=1}^{N_{s}} \rho D_{j} \frac{\partial Y_{j}}{\partial x_{\alpha}},
$$

which is capable to account for differential diffusion. $D_{i}$ denotes the diffusion coefficient of the species $i$. The correction of Coffee and Heimerl [26] (second term in Eq. (3)) is applied to ensure that the sum of the diffusive mass fluxes vanishes. The diffusive heat flux is given by superposition of the terms resulting from Fourier's law of heat conduction and the diffusive fluxes of the enthalpy [27]

$$
q_{\alpha}=-\lambda \frac{\partial T}{\partial x_{\alpha}}+\sum_{i=1}^{N_{s}} h_{i} j_{i \alpha}
$$

$\lambda$ is the thermal conductivity of the gas mixture and $h_{i}$ the specific enthalpy of the $i$-th species. The conditional expectations of these fluxes are decomposed into a Favre average and a fluctuating part [16, 18], i.e.

$$
\begin{aligned}
& \sum_{\alpha=1}^{3}\left\langle\frac{1}{\rho} \frac{\partial j_{i \alpha}}{\partial x_{\alpha}} \mid \boldsymbol{\psi}\right\rangle=\sum_{\alpha=1}^{3} \widehat{\frac{1}{\rho} \frac{\partial j_{i \alpha}}{\partial x_{\alpha}}}+\sum_{\alpha=1}^{3}\left\langle\left(\frac{1}{\rho} \frac{\partial j_{i \alpha}}{\partial x_{\alpha}}\right)^{\prime \prime} \mid \boldsymbol{\psi}\right\rangle, \\
& \sum_{\alpha=1}^{3}\left\langle\frac{1}{\rho} \frac{\partial q_{\alpha}}{\partial x_{\alpha}} \mid \boldsymbol{\psi}\right\rangle=\sum_{\alpha=1}^{3} \frac{1}{\rho} \frac{\partial q_{\alpha}}{\partial x_{\alpha}}+\sum_{\alpha=1}^{3}\left\langle\left(\frac{1}{\rho} \frac{\partial q_{\alpha}}{\partial x_{\alpha}}\right)^{\prime \prime} \mid \boldsymbol{\psi}\right\rangle
\end{aligned}
$$

where $\tilde{\bullet}$ denotes the Favre average and $\bullet$ " the fluctuation. The Favre averaged contributions to the mixing term are simplified by

$$
\widehat{\frac{1}{\rho} \frac{\partial j_{i \alpha}}{\partial x_{\alpha}}}=\frac{1}{\langle\rho\rangle} \frac{\partial\left\langle j_{i \alpha}\right\rangle}{\partial x_{\alpha}}, \quad \widehat{\frac{1}{\rho} \frac{\partial q_{\alpha}}{\partial x_{\alpha}}}=\frac{1}{\langle\rho\rangle} \frac{\partial\left\langle q_{\alpha}\right\rangle}{\partial x_{\alpha}}
$$


and the expectations of the diffusive fluxes are evaluated by invoking a formulation corresponding the to molecular fluxes of Eqs. (3) and (4) [27], i.e.

$$
\begin{aligned}
& \left\langle j_{i \alpha}\right\rangle \approx-\langle\rho\rangle\left\langle D_{i}\right\rangle \frac{\partial \tilde{Y}_{i}}{\partial x_{\alpha}}+\tilde{Y}_{i} \sum_{j=1}^{N_{s}}\langle\rho\rangle\left\langle D_{j}\right\rangle \frac{\partial \tilde{Y}_{j}}{\partial x_{\alpha}}, \\
& \left\langle q_{\alpha}\right\rangle \approx-\langle\lambda\rangle \frac{\partial \tilde{T}}{\partial x_{\alpha}}+\sum_{i=1}^{N_{s}} \tilde{h}_{i}\left\langle j_{i \alpha}\right\rangle .
\end{aligned}
$$

Under these simplifications the Favre averaged diffusive fluxes appear in closed form in the MDF transport equation. They are, however, usually ignored in transported PDF methods, if high Reynolds numbers are assumed [16]. In this form, the model equations are similar to the results of McDermott et al. [18]. This model approach, however, is applicable to RANS. In the RANS context, where all turbulent scales are modeled, a closure is required for the conditional fluctuations. The contributions of the fluctuating terms in Eqs. (5) and (6) are modeled by the IEM model [2] yielding

$$
\begin{aligned}
\sum_{\alpha=1}^{3}\left\langle\left(\frac{1}{\rho} \frac{\partial j_{i \alpha}}{\partial x_{\alpha}}\right)^{\prime \prime} \mid \psi\right\rangle & =\frac{1}{2} C_{\phi} \omega\left(Y_{i}-\tilde{Y}_{i}\right), \\
\sum_{\alpha=1}^{3}\left\langle\left(\frac{1}{\rho} \frac{\partial q_{\alpha}}{\partial x_{\alpha}}\right)^{\prime \prime} \mid \psi\right\rangle & =\frac{1}{2} C_{\phi} \omega(h-\tilde{h}) .
\end{aligned}
$$

In the above equations $\omega$ denotes the turbulent frequency and $C_{\phi}$ the mechanical-to-scalar timescale ratio. With these equations the conditional diffusive fluxes are closed. Defining the diffusion velocities

$$
\tilde{V}_{Y i}=-\sum_{\alpha=1}^{3} \frac{1}{\langle\rho\rangle} \frac{\partial\left\langle j_{i \alpha}\right\rangle}{\partial x_{\alpha}}, \quad \tilde{V}_{h}=-\sum_{\alpha=1}^{3} \frac{1}{\langle\rho\rangle} \frac{\partial\left\langle q_{\alpha}\right\rangle}{\partial x_{\alpha}},
$$


finally yields

$$
\begin{aligned}
& -\sum_{\alpha=1}^{3}\left\langle\frac{1}{\rho} \frac{\partial j_{i \alpha}}{\partial x_{\alpha}} \mid \boldsymbol{\psi}\right\rangle=\tilde{V}_{Y i}-\frac{1}{2} C_{\phi} \omega\left(Y_{i}-\tilde{Y}_{i}\right), \\
& -\sum_{\alpha=1}^{3}\left\langle\frac{1}{\rho} \frac{\partial q_{\alpha}}{\partial x_{\alpha}} \mid \boldsymbol{\psi}\right\rangle=\tilde{V}_{h}-\frac{1}{2} C_{\phi} \omega(h-\tilde{h}) .
\end{aligned}
$$

With this formulation of the mixing term, the transition of turbulent to molecular mixing is included in RANS/transported PDF methods. The model is formally similar to the approach introduced by McDermott et al. [18] Both approaches are capable to recover a laminar flow solution, provided that the residual stress tensor (in case of the LES) and the Reynolds stress tensor (in case of the RANS) vanish. There is, however, a difference between these models, since the approach of McDermott et al. [18] reproduces the transition of LES/FDF computations into a DNS for vanishing LES filter width. This is not possible in a RANS where all turbulent scales are modeled.

The effect of this diffusion model on the MDF is understood by studying the following simplified MDF evolution equation (Einstein notation)

$$
\frac{\partial \mathscr{F}_{\phi}}{\partial t}+\frac{\partial}{\partial \psi_{k}}\left(\tilde{V}_{\phi k} \mathscr{F}_{\phi}\right)=0
$$

Since the mean diffusion velocities do not depend on the state variables $\boldsymbol{\psi}$ the above equation is simplified reading (Einstein notation)

$$
\frac{\partial \mathscr{F}_{\phi}}{\partial t}+\tilde{V}_{\phi k} \frac{\partial \mathscr{F}_{\phi}}{\partial \psi_{k}}=0
$$

This partial differential equation is solved easily with the methods of characteristics [28] yielding

$$
\mathscr{F}_{\phi}\left(\psi\left(t ; \boldsymbol{\psi}_{0}\right), t\right)=\mathscr{F}_{\phi 0}
$$


The MDF is transported in composition space through diffusion along the characteristic $\boldsymbol{\psi}\left(t ; \boldsymbol{\psi}_{0}\right)$ and its initial shape $\mathscr{F}_{\phi 0}=\mathscr{F}_{\phi}\left(\boldsymbol{\psi}_{0}, t_{0}\right)$ is preserved. To address the effect of the present approach on the means, Eq. (9) is multiplied with the sample space variable and integrated over composition space. The results is

$$
\frac{\partial \tilde{\phi}_{i}}{\partial t}=\tilde{V}_{\phi i} .
$$

Hence, the Favre average of the thermochemical variables is altered at a rate given by the Favre averaged diffusion term as noted by McDermott et al. [18]. The temporal evolution of covariances is derived in the same manner by multiplying Eq. (9) with the product of Favre fluctuation and taking the expectation. This yields

$$
\frac{\partial}{\partial t}\left(\widetilde{\phi_{i}^{\prime \prime} \phi_{j}^{\prime \prime}}\right)=0 .
$$

Thus, Favre averaged diffusion does not affect the covariances in this model. The change in covariances is solely modeled by the IEM model in Eqs. (7) and (8).

\section{Particle method}

Using the models described in the previous section the modeled MDF transport equation is given by (Einstein notation)

$$
\begin{aligned}
& \frac{\partial \mathscr{F}_{\phi}}{\partial t}+\frac{\partial}{\partial x_{\alpha}}\left(\left(\tilde{u}_{\alpha}+\frac{1}{\langle\rho\rangle} \frac{\partial\left(\langle\rho\rangle D_{T}\right)}{\partial x_{\alpha}}\right) \mathscr{F}_{\phi}\right)-\frac{\partial^{2}}{\partial x_{\alpha} \partial x_{\alpha}}\left(D_{T} \mathscr{F}_{\phi}\right) \\
& +\frac{\partial}{\partial h}\left(\left(-\frac{C_{\phi}}{2} \omega(h-\tilde{h})+\tilde{V}_{h}\right) \mathscr{F}_{\phi}\right) \\
& +\frac{\partial}{\partial Y_{i}}\left(\left(\frac{\dot{m}_{i}}{\rho}-\frac{C_{\phi}}{2} \omega\left(Y_{i}-\tilde{Y}_{i}\right)+\tilde{V}_{Y i}\right) \mathscr{F}_{\phi}\right)=0 .
\end{aligned}
$$


In order to solve the equation a hybrid finite-volume/Lagrangian MonteCarlo method [24] is implemented in the THETA code of the German Aerospace Center [29]. The Lagrangian Monte-Carlo solver solves numerically the following system of stochastic differential equations (SDE)

$$
\begin{aligned}
& x_{\alpha}^{*}(t+d t)=x_{\alpha}^{*}(t)+\left(\tilde{u}_{\alpha}+\frac{1}{\langle\rho\rangle} \frac{\partial}{\partial x_{\alpha}}\left(\langle\rho\rangle D_{T}\right)\right) d t+\sqrt{2 D_{T}} d W_{\alpha}, \\
& h^{*}(t+d t)=h^{*}(t)+\left(-\frac{C_{\phi}}{2} \omega\left(h^{*}(t)-\tilde{h}\right)+\tilde{V}_{h}\right) d t, \\
& Y_{i}^{*}(t+d t)=Y_{i}^{*}(t)+\left(\frac{\dot{m}_{i}}{\rho}-\frac{C_{\phi}}{2} \omega\left(Y_{i}^{*}(t)-\tilde{Y}_{i}\right)+\tilde{V}_{Y i}\right) d t,
\end{aligned}
$$

which govern the evolution of stochastic particles. $W_{\alpha}$ denotes a Wiener process (which is the stochastic force in these SDEs), and "*" quantities in the stochastic system. The PDF of this stochastic system provides a model for the Lagrangian PDF of the flow. The latter one is the transition density of the MDF as demonstrated by Pope [24]. In this way it is ensured that the MDF of the stochastic system is equivalent to the MDF of the reactive flow provided that the consistency condition

$$
\langle\rho(\boldsymbol{x})\rangle=\left\langle\sum_{n=1}^{N_{P}} m_{n} \delta\left(\boldsymbol{x}-\boldsymbol{x}^{(n)}\right)\right\rangle
$$

holds [24]. $m_{n}$ denotes the mass of a stochastic particle, $\delta$ the Dirac delta function, and $N_{p}$ the number of stochastic particles representing the MDF. With this system of stochastic particles, the Favre average $\tilde{\phi}_{k}$ of an arbitrary thermochemical quantity is estimated from the properties $\phi_{k}^{*(n)}$ of the stochastic particles by

$$
\tilde{\phi}_{k} \approx \frac{\sum_{n=1}^{N_{p \mathrm{VOL}}} m_{n} \phi_{k}^{*(n)}}{\sum_{n=1}^{N_{p \mathrm{VOL}}} m_{n}} .
$$


This estimate is valid for a small, statistically homogenous volume in physical space [24] and includes the summation over $N_{p \text { VOL }}$ particles present in the volume. For the solution of the system of coupled SDEs the method of fractional steps [24] is used, which is equivalent to a factorization of the MDF transport equation. For molecular diffusion, a separate fractional step is introduced. As proposed by McDermott et al. [18] the mean diffusion equations (12) are solved for enthalpy and composition, i.e.

$$
\begin{gathered}
\frac{\partial \tilde{h}}{\partial t}=\tilde{V}_{h}, \\
\frac{\partial \tilde{Y}_{i}}{\partial t}=\tilde{V}_{Y i} .
\end{gathered}
$$

The resulting change in Favre averaged enthalpy and mass fraction, i.e. $\Delta \tilde{h}^{(n)}$ and $\Delta \tilde{Y}_{i}^{(n)}$, is directly imposed on the stochastic particles [18] yielding

$$
\begin{aligned}
& Y_{i}^{*(n+1)}=Y_{i}^{*(n)}+\Delta \tilde{Y}_{i}^{(n)}, \\
& h^{*(n+1)}=h^{*(n)}+\Delta \tilde{h}^{(n)} .
\end{aligned}
$$

The same correction is used for all particles in a cell. This approach is known as "Local Constant Mean Estimate" (LCME), which satisfies both local and global conservation of the mean [16]. Provided that the grid resolution is chosen fine enough for the problem at hand, this approach is justified since the cell averages vary only moderately across a cell [16]. For the solution of Eqs. (20) and (21) the finite-volume method is used. The fluxes on the cell faces are evaluated using central differences with second order accuracy in space. $\tilde{V}_{h}$ and $\tilde{V}_{Y i}$ are then determined at the cell centers using Gauss' divergence theorem. For the evaluation of the gradients time averaged values of mass fractions and temperature are used, in order to avoid stochastic 
"noise". This is possible, since all results discussed in this work are obtained from steady state computations. For discretization of Eqs. (20) and (21) in time a first order explicit Euler scheme is used, where the time step $\Delta t_{D}$ stemming from diffusion is limited to [30]

$$
\begin{aligned}
\Delta t_{D}= & \frac{1}{2}\left(\left(\sum_{\alpha=1}^{3} \frac{1}{\Delta x_{\alpha}^{2}}\right) \max \left(\left\langle\lambda_{k}\right\rangle /\left(\langle\rho\rangle_{k}\left\langle c_{p, k}\right\rangle\right),\left\langle D_{i, k}\right\rangle\right)\right)^{-1} \\
& k=1, . ., N_{\text {cell }} ; i=1, \ldots, N_{s}
\end{aligned}
$$

in order to ensure stability. Here $\Delta x_{\alpha}$ denotes the extensions of the cell in the three spatial dimensions. The maximum of the transport coefficients is taken over all cells in a grid with a total of $N_{\text {cell }}$ finite-volume cells in order to avoid stochastic "noise" in the local time steps at the beginning of the calculation. In addition the Courant-Friedrich-Lewy (CFL) criterion must be satisfied for the solution of Eq. (15) given by [31]

$$
\Delta t_{T}=\left(\sum_{\alpha=1}^{3} \frac{1}{\Delta x_{\alpha}}\left|\tilde{u}_{\alpha}+\frac{1}{\langle\rho\rangle} \frac{\partial\left(\langle\rho\rangle D_{T}\right)}{\partial x_{\alpha}}\right|+\frac{2 D_{T}}{\sum_{\beta=1}^{3} \Delta x_{\beta}^{2}}\right)^{-1} C F L .
$$

The $C F L$ number is set to 0.7 in this work. The time steps $\Delta t_{D}$ and $\Delta t_{T}$ are then combined to the integration time step [30]

$$
\Delta t=\left(\frac{1}{\Delta t_{D}}+\frac{1}{\Delta t_{T}}\right)^{-1} .
$$

This time step limitation is necessary for the stability of the numerical scheme. A major problem is to enforce the boundedness of mass fractions in Eq. (22). This stems from the fact, that the present scheme does not ensure strong realizability [24] on the level of stochastic particles. To solve this problem, McDermott et al. [18] propose a limitation of the turbulent frequency 
$\omega$ in the IEM model. One disadvantage of this approach is, however, that errors occurring from boundedness violations are balanced by an artificial, unphysical increase of the mixing rate. Due to this drawback, this approach is not used here and another method is employed to enforce boundedness. Firstly, the diffusion step is followed directly by the IEM mixing step, which relaxes the particle properties towards the bounded Favre mean. Secondly, the time step is limited such that changes through diffusion remain small during the numerical solution procedure. It turns out that the time step limit according to Eq. (26) is sufficient to ensure boundedness. In this way an artificial increase of $\omega$ is avoided.

The closure of Eqs. (15) to (17) requires the turbulence time and length scales to determine $\omega$ and $D_{T}$. To this end turbulence model equations are solved along with the Reynolds averaged Navier-Stokes equations and a pressure correction equation in the finite-volume solver. Turbulence time and length scales are modeled using the standard $k-\varepsilon$ model [32] or the $k$ - $\omega$ model of Wilcox [33], depending on the test case. For the solution of these equations the finite-volume solver receives the expectations of dynamic viscosity and density. Both values are computed using Eq. (19), where the variables are weighted with the specific volumes to retrieve expectations instead of Favre averages. All averages are calculated as "Cloud-in-Cell" (CIC) mean [16], i.e. averaging includes all particles in a given cell. Since the averaging over a finite number of particles introduces a stochastic error, time averaging is performed additionally for steady state calculations. The algorithm used is described by Wang et al. [34] in more detail. During the calculation data are 
exchanged between the solvers after each iteration. This is known as tight coupling [35].

\section{Heat transfer modeling}

Two different heat transfer problems are presented in this section. The first one involves the heat transfer in a two dimensional channel and serves as a verification test case for the diffusion model. The second test case concerns the thermal entrance region in a turbulent flow. Experimental data are provided here which help to validate the model. All model calculations presented are at steady state.

\subsection{Two dimensional channel}

The model approach for the mixing term is verified first by the computation of a heat transfer problem in a two dimensional channel flow. One of the channel walls is kept at a constant temperature of $100^{\circ} \mathrm{C}$ whereas the second wall is adiabatic. Air enters the channel at a temperature of $20^{\circ} \mathrm{C}$ and a pressure of $100 \mathrm{kPa}$. The velocity is chosen such that the channel height based Reynolds number equals 10,000 (the channel height is $10 \mathrm{~mm}$ ). The channel is discretized with 25,000 hexahedral cells. To model turbulence the $k-\omega$ turbulence model Wilcox is used [33]. The MDF is represented by an ensemble of 64 stochastic particles per cell. For an assessment of the model, reference data are required. These data are provided by a "numerical experiment", i.e. a RANS computation of the flow, which is performed using the same turbulence model. In this RANS computation, the mean temperature follows from the averaged energy conservation equation. Computational results of this study are given in Fig. 1 at a distance of $0.48 \mathrm{~m}$ from the channel 
inlet. In order to resolve the near-wall region, the wall distance is plotted on a logarithmic scale in the right diagram. The comparison between the RANS computation and the transported PDF calculation employing the diffusion model (curve "TPDF-DIFF") reveals that the PDF method produces the same results as the RANS model. The heat fluxes through the wall at this position computed by the two different methods also agree very well. It amounts to $601 \mathrm{~W} / \mathrm{m}^{2}$ in case of the transported PDF and $607 \mathrm{~W} / \mathrm{m}^{2}$ in case of the RANS computation respectively, which corresponds to $1 \%$ difference. In order to assess the importance of the diffusion model, the transported PDF computations are repeated with the diffusion velocities in Eqs. (7) and (8) set to zero. This is equivalent to invoking the high Reynolds number assumption which is usually used in transported PDF methods. The computational results prove that the IEM model alone is not able to model the heat transfer correctly. Using the IEM model the temperature stays almost constant across the whole channel and rises sharply close to the wall (curve "TPDFIEM"). This behavior is understood best in the light of the particle method. All mixing models, which neglect molecular transport, change the variance

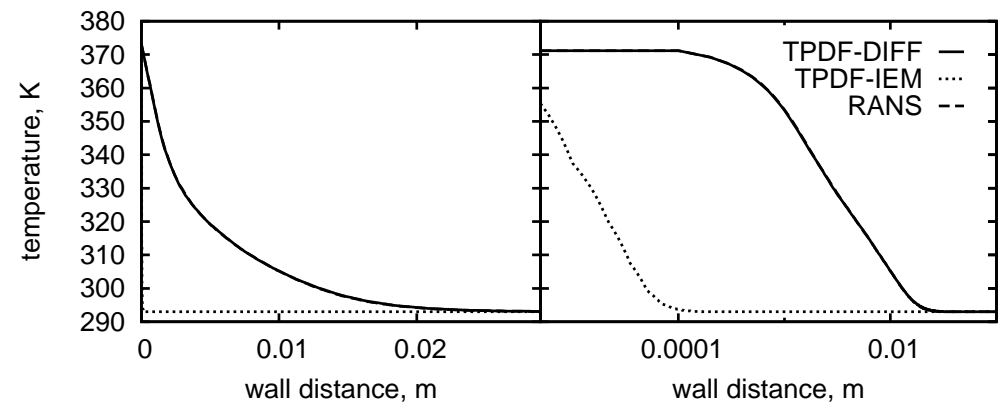

Figure 1: Favre averaged temperature in a turbulent channel flow. 
only and keep the mean constant [16]. Then the only way to transport heat away from the wall and to achieve a change in the means is to move heated particles from the wall towards the center of the channel. The main flow direction, however, is parallel to the wall. Therefore, the only way to accomplish a wall-normal spatial transport is the Wiener process in Eq. (15). Its intensity depends on the turbulent diffusivity which vanishes as the wall is approached. Thus the heated particles remain trapped close to the wall and no wall normal transport of heat is accomplished. The inclusion of averaged diffusive fluxes according to Eqs. (7) and (8) (in the present case of averaged heat conduction) is therefore important for modeling wall bounded flows with transported PDF methods.

\subsection{Thermal entrance region in a turbulent flow}

In a second step the diffusion model is applied in a calculation of the thermal entrance region in a fully developed, turbulent pipe flow, which has been investigated experimentally by Abbrecht and Churchill [20]. In this test case air at a temperature of $299.5 \mathrm{~K}$ is fed at an average velocity of $6.287 \mathrm{~m} / \mathrm{s}$ into a straight pipe of 1.52 inch $(38.608 \mathrm{~mm})$ diameter, which corresponds to a diameter based Reynolds number of 15,000. This air runs first through an unheated section of 66 inch $(1676.4 \mathrm{~mm})$ length in order to achieve a fully developed pipe flow. After this section the wall is heated to reach a temperature of $313.2 \mathrm{~K}$. Within this heated section Abbrecht and Churchill [20] provide data for the radial distribution of the dimensionless temperature

$$
\vartheta=\frac{T-T_{0}}{T_{W}-T_{0}}
$$




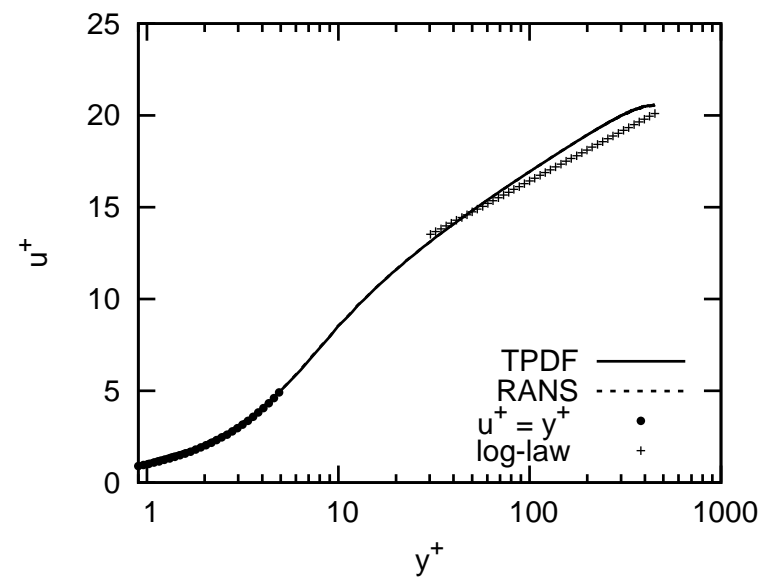

Figure 2: Axial flow velocity profiles in the pipe.

which is defined in terms of the wall temperature $T_{W}$ and the bulk temperature $T_{0}$. The temperatures are measured at $0.453,1.13,4.12$, and 9.97 diameters distance in the thermal entrance region. For the computations a $10^{\circ}$ azimuthal segment of the pipe is considered due to the symmetry of the problem. The domain is discretized with 9,900 cells. As in subsection 4.1, the heat transfer is not only modeled with the transported PDF method, but also by solving the Favre averaged energy conservation equation, in order to provide a reference for the transported PDF calculations. Turbulence is modeled by the $k-\omega$ turbulence model [33] and the turbulent Prandtl number is set to 0.9, as recommended by Malhotra and Kang [36]. For the discrete representation of the MDF 64 stochastic particles are used per cell. In Fig. 2 the calculated dimensionless velocity profiles of the RANS and the transported PDF method are given at a distance of $10 \mathrm{~mm}$ upstream of the thermal entrance. Both methods yield identical results. Comparing the computations to theoretical velocity profiles in the viscous sublayer and 

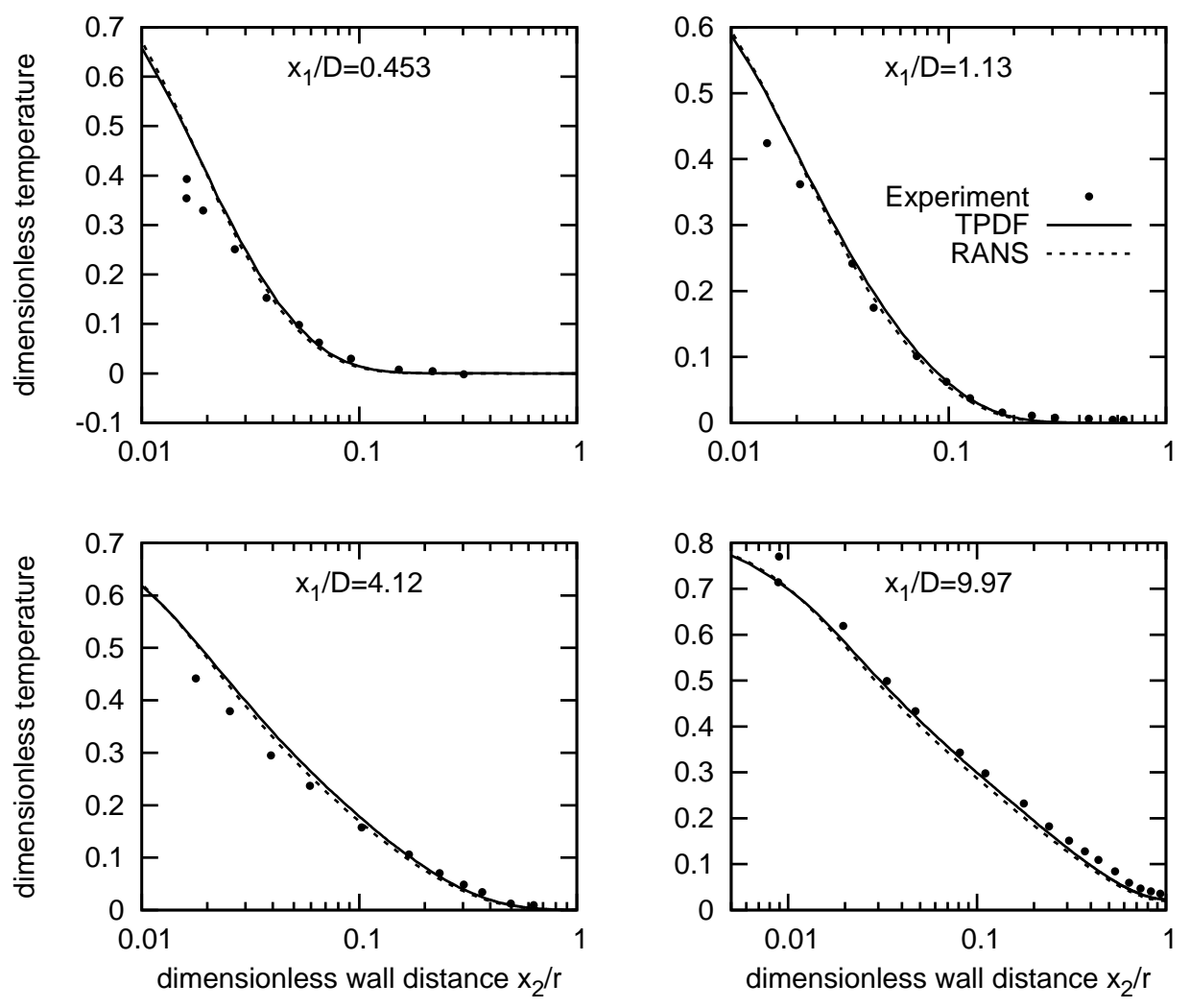

Figure 3: Dimensionless temperature in thermal entrance region of the pipe.

the log-law region [37] shows, that the flow is fully developed at this point. In Fig. 3 the non-dimensional temperature is plotted as a function of the wall distance, which is normalized by the pipe radius $r$. Experimental data and computational results of the RANS and the transported PDF method are shown. Between the RANS and the transported PDF method no differences are observed, which confirms the findings in subsection 4.1. The general agreement between calculation and experimental data is excellent. Some minor differences are observed at $x_{1} / \mathrm{D}=0.453$ at $x_{2} / r=0.017$ and at $x_{1} / \mathrm{D}=4.12$ between $x_{2} / r=0.01$ and $x_{2} / r=0.04$. The diffusion model, 
however, proves capable to predict heat transfer with the same accuracy as RANS methods.

\section{Flame calculation}

The diffusion model is applied next in transported PDF computations of a turbulent hydrogen-air flame ("H3 flame"). This flame is non-premixed and is operated without a stabilizing pilot burner. The fuel jet consisting of 50 vol.- $\%$ nitrogen and 50 vol.- $\%$ hydrogen is issued at an average flow velocity of $34.8 \mathrm{~m} / \mathrm{s}$ through a nozzle with $8 \mathrm{~mm}$ diameter into the ambient air. The diameter based Reynolds number of the fuel jet is 10,000. To provide an uniform flow environment, the flame is surrounded by an air coflow $(0.2 \mathrm{~m} / \mathrm{s}$ flow velocity) of $140 \mathrm{~mm}$ diameter. Details on the arrangement are given in $[21,22]$. Experimental data for temperature and species concentrations are given in $[22,38]$ whereas measurements of flow velocities are summarized in [39].

\subsection{Computational setup}

The rotational symmetry of the burner is exploited in order reduce computational cost. The flame is computed on a domain consisting of a $10^{\circ} \mathrm{az}-$ imuthal segment of the burner. As in the case of the pipe flow, steady state calculations are performed. To ensure the numerical accuracy four cases with different discretizations in terms of mesh size and particle number are investigated (see Tab. 1). Cases 1 and 2 utilize the same mesh with 17,250 cells but differ in particle number. The smallest particle number is employed by case 1 with 64 particles per cell, the largest by case 2 with 256 particles per cell. In case 3 and case 4 the particle number is kept at 64 particles and 
Table 1: Computational cases for the "H3 Flame".

case number of grid cells number of stochastic particles per cell

\begin{tabular}{lll}
\hline 1 & 17,250 & 64 \\
2 & 17,250 & 256 \\
3 & 35,333 & 64 \\
4 & 186,484 & 64
\end{tabular}

the mesh size is increased to 35,333 and 186,484 cells in order to study the grid dependency of the solution. The meshes of cases 1 to 3 provide the finest resolution close to the nozzle exit plane, where the cell growth rate in axial direction is 1.05. During mesh refinement from case 1 to case 3 the mesh resolution is refined mainly in radial direction. Thus, the coarse mesh of the cases 1 and 2 provides 15 and the fine mesh of case 323 cells across the radius of the fuel pipe. A corresponding refinement is employed in the region of the shear layer of the turbulent jet. It is also important to relate the mesh resolution at the flame root to local diffusion length scales, since mixing is controlled by molecular diffusion in this area. As demonstrated later in Fig. 11 the temperature data close to the nozzle exit (at $x_{1} / \mathrm{D}=2.5$ ) correlate with those of a laminar diffusion flame at a strain rate of $a=181 / \mathrm{s}$. Using this strain rate together with the diffusion coefficient at stoichiometric conditions, $D_{s t}$, the diffusion length scale $l_{D}$ may be estimated by [40]

$$
l_{D}=\left(\frac{D_{s t}}{a}\right)^{1 / 2} \text {. }
$$


For the given conditions a length scale of $l_{D}=6 \mathrm{~mm}$ is obtained. The coarse and the fine meshes have cell spacings in the order of $100 \mu \mathrm{m}$ and $200 \mu \mathrm{m}$, respectively, in this region. Based on this estimate for the diffusion length scale, there are 60 volumes of the fine mesh and 30 of the coarse mesh to resolve the flame. This mesh size is still coarse in relation to flame thickness. For this reason in case 4 the mesh is locally adapted in the shear layer yielding cell sizes of $12.5 \mu \mathrm{m}$. Thus, the mesh used in case 4 is one order of magnitude finer than the mesh in case 3. The oxidation of hydrogen is described by the reaction mechanism of Peters and Rogg [41]. To account for nitric oxide formation this reaction mechanism is combined with the NO reaction subset of the San Diego mechanism [42] which provides sufficient accuracy in NO computations [43]. For the modeling of turbulence the two equation standard $k-\varepsilon$ model of Jones and Launder [32] is used with the model constants of Launder and Sharma [44] except for the $C_{\varepsilon 1}$ model constant which is increased to a value of $C_{\varepsilon 1}=1.53$. In this way the round jet anomaly of the $k-\varepsilon$ model is corrected. In order to model the inflow of the fuel jet into the surroundings correctly, the last section of the pipe internal flow (50 diameters length) is fully included into the computational domain. This length is sufficient to obtain a developed pipe flow. At the pipe inlet a turbulence intensity of $5 \%$ is prescribed and a length scale of $0.56 \mathrm{~mm}$ which corresponds to $7 \%$ of the pipe diameter [45]. The model constant of the IEM model, the mechanical-to-scalar timescale ratio is $C_{\phi}=2$ [24]. In all calculations the described diffusion model is employed. Without this model a calculation of the flame could not be accomplished. Using only the IEM model to close conditional diffusive fluxes in the MDF transport equation 
results in local extinction at the nozzle exit. Next, the flame lifts off until global extinction occurs. Increasing the mechanical-to-scalar timescale ratio does not solve the problem. A stable flame is only obtained if the diffusion model is active. This behavior is not surprising. Meier et al. [22] report that "in this region, the flame exhibits many of the characteristics of a laminar flow and the different molecular species are not mainly transported and mixed by turbulence but also by molecular diffusion". Therefore, mixing models which are based on a mixing rate proportional to the turbulent frequency $\omega$ fail in areas, where the flow is essentially laminar. Mixing of reactants is provided here by the drift in composition space stemming from the diffusion model.

\subsection{Flame structure}

Computational results for Favre averaged temperature, species mass fractions, and axial flow velocities are compared with experimental data in Fig. 4 along the flame axis. The axial coordinate is non-dimensionalized with the nozzle diameter of $8 \mathrm{~mm}$. All computational cases of Tab. 1 are evaluated here. The differences between these results are small. Therefore, the resolution is sufficient to provide a solution which is nearly independent of mesh size and particle number.

A comparison of the computational and experimental data for temperature in Fig. 4 shows that the flame is wider in the calculation than in the experiment. These deviations are an artefact of the round jet correction applied here. This correction is capable to produce the correct jet spreading rate in the near field but fails beyond 40 diameters distance. The radial profiles of axial flow velocity confirm this. In Fig. 5 axial flow velocities are 
given at five different axial positions. In the near field up to a distance of 40 diameters the mean axial velocity is predicted correctly. Further downstream at $x_{1} / \mathrm{D}=60$ and $x_{1} / \mathrm{D}=80$, however, the calculated axial velocities spread less than in the measurement. The overall profile is narrower in the calculation. The smaller spreading in the calculation yields a reduced entrainment of air which reduces in turn the mixing of air and exhaust gases. Thus, the temperature in the exhaust gases remains too high. Reducing the $C_{\varepsilon 1}$ model constant does not solve the problem. Previous model calculations of the same flame with a model constant of $C_{\varepsilon 1}=1.44$ demonstrate that in this case the field beyond 40 diameters is calculated correctly, the near field, however, suffers from an overestimation of spreading rate [29]. In the light of these modeling problems, the overall agreement between the thermochemical variables is good. The profiles of Favre averaged temperature and major species mass fractions, i.e. $\mathrm{H}_{2}, \mathrm{H}_{2} \mathrm{O}, \mathrm{O}_{2}$ and $\mathrm{N}_{2}$, match the experimental data well. In the minor species $\mathrm{OH}$ and $\mathrm{NO}$, however, the measured data are underestimated by approximately $25 \%$. For nitric oxides it is known, that this deviation stems from inaccuracies in the reaction mechanism [43]. For the same thermochemical variables the rms values are evaluated from the transported PDF calculation. Results along the flame axis are summarized in Fig. 6. The rms values are computed for case 2 only since it employs the largest number of stochastic particles. In contrast to the first moments, the rms values are not time averaged, which explains the stochastic noise in these profiles. In general, the agreement between measured and calculated rms values is good. Some deviations are observed in the rms of temperature and the rms of mass fraction of $\mathrm{H}_{2} \mathrm{O}$ and $\mathrm{O}_{2}$ which are all underestimated 


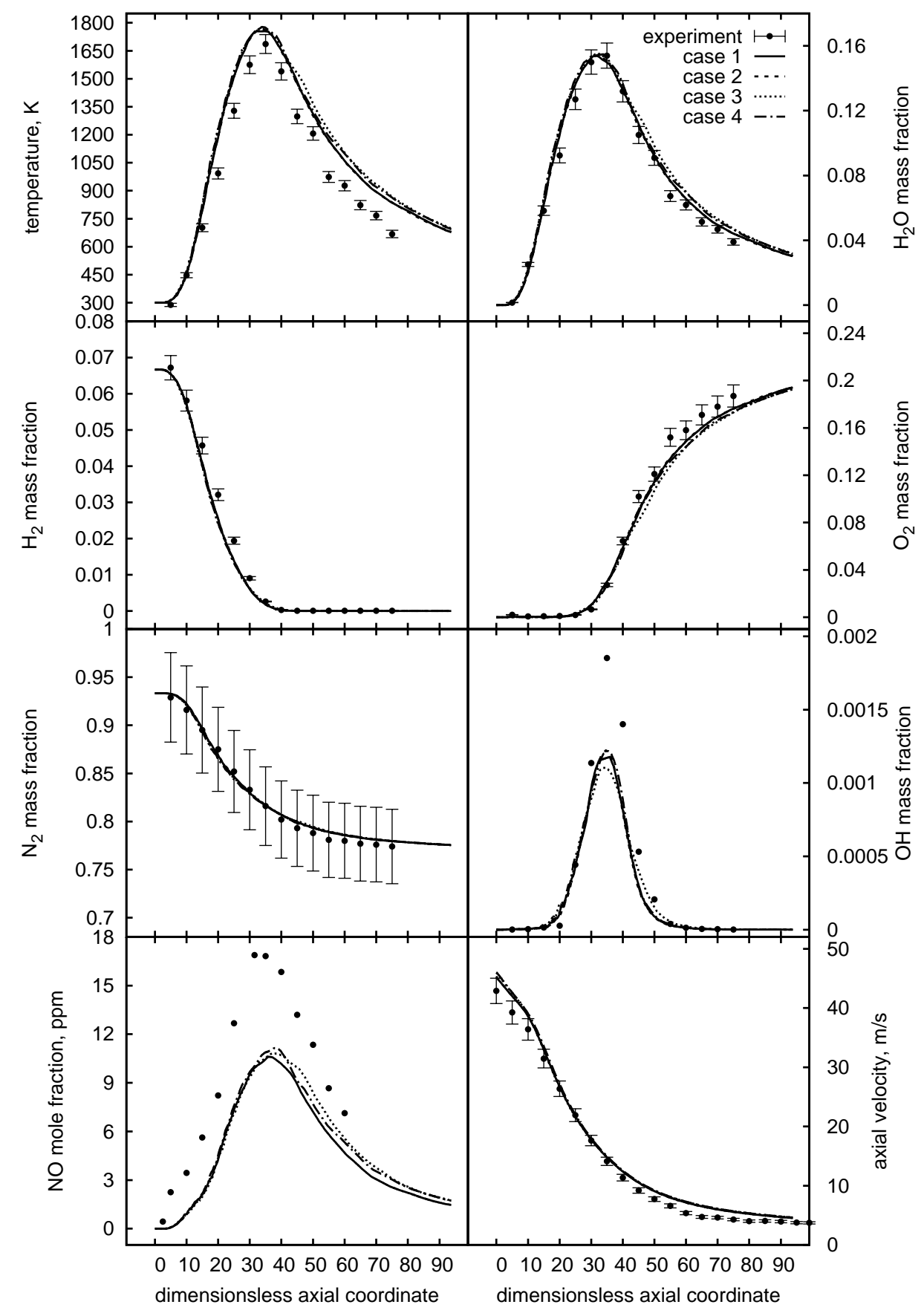

Figure 4: Favre averages along flame axis. 

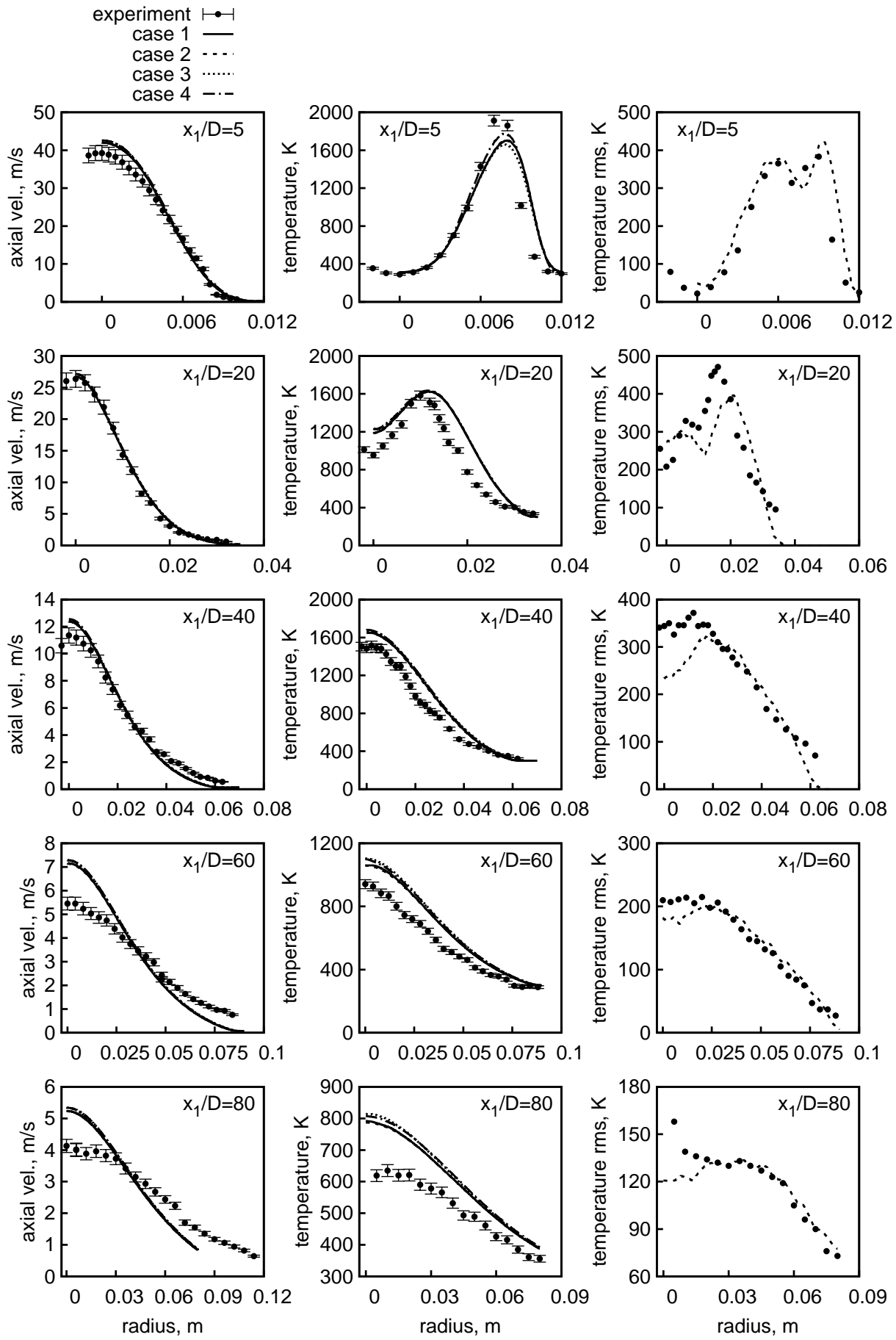

Figure 5: Radial distribution of flow quantities at different locations. 


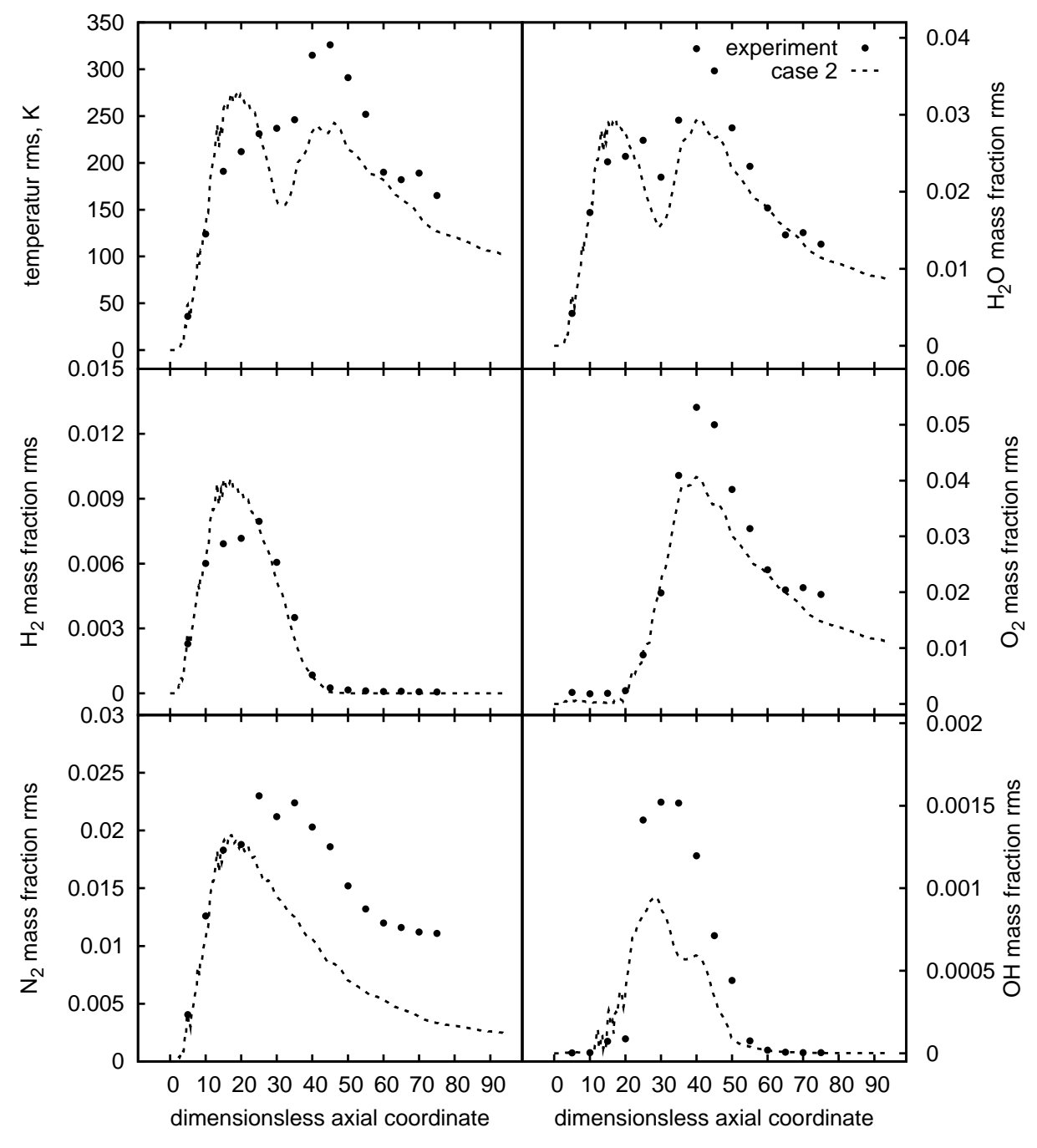

Figure 6: RMS values along flame axis. 
between 40 and 60 diameters distance. The mass fraction rms for $\mathrm{N}_{2}$ and $\mathrm{OH}$ deviates after a distance of 30 diameters significantly from the measurements, which is caused by a poor prediction of the macroscopic mixing in this area. A measure of the mixedness are the gradients of the Favre averaged thermochemical variables, since steep gradients correspond to a high degree of unmixedness. In the region, where the deviations between experimental data and model calculation are observed, calculated gradients in the Favre averages of these variables are not as steep as in the experiment. Thus, the calculated turbulent production of variance, which is proportional to the gradients of the Favre averages [16], is smaller compared to the experiment and, therefore, smaller rms values are found. Consistently the computation overestimates the rms values for variables, whose calculated Favre averaged profiles are steeper compared to the experiments. This is the case for the mass fractions of $\mathrm{H}_{2}$.

These problems in the estimation of the flame properties are also reflected in the radial distribution of the thermochemical variables. In Fig. 5 the radial profiles of Favre averaged temperature and temperature rms are shown. The radial agreement between calculation and experiment is good in areas where the axial profile is well reproduced. Favre averaged temperatures agree well with experimental data up to a distance of 40 diameters. Further downstream the Favre averaged temperature is overestimated as already observed in the axial profile. The temperature rms agrees very well at all axial positions with exception of the profile at 40 diameters distance, where the axial value at $r=0 \mathrm{~m}$ is underestimated. Deviations at this position are also observed in 


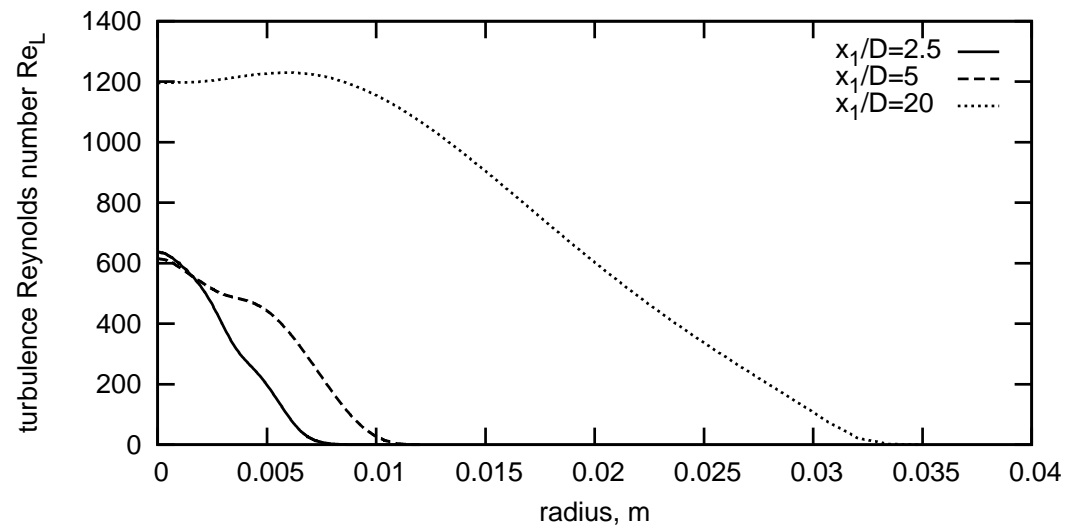

Figure 7: Turbulence Reynolds number $R e_{L}$.

the axial profile of temperature rms.

\subsection{Analysis of turbulent and molecular transport}

As discussed earlier the inclusion of Favre averaged diffusion is important to stabilize the flame. It is therefore interesting to investigate the relative importance of turbulent and molecular transport in the "H3 flame". Hence, in a first step the local turbulence Reynolds number [16]

$$
R e_{L}=\frac{k^{2}}{\varepsilon \nu},
$$

is analyzed, which is defined in terms of the integral length scale $k^{3 / 2} / \varepsilon$, the characteristic velocity $k^{1 / 2}$ and the molecular viscosity $\nu$. It may be interpreted as the Reynolds number of the largest eddies in the flow [37]. Computational results for the "H3 flame" are given in Fig. 7 for case 2, where $R e_{L}$ is evaluated as a function of the radius at $x_{1} / \mathrm{D}=2.5,5$, and 20. The smallest turbulence Reynolds numbers are found close to the nozzle at $x_{1} / \mathrm{D}=2.5$ with a maximum value of $R e_{L} \approx 600$. At this position high 

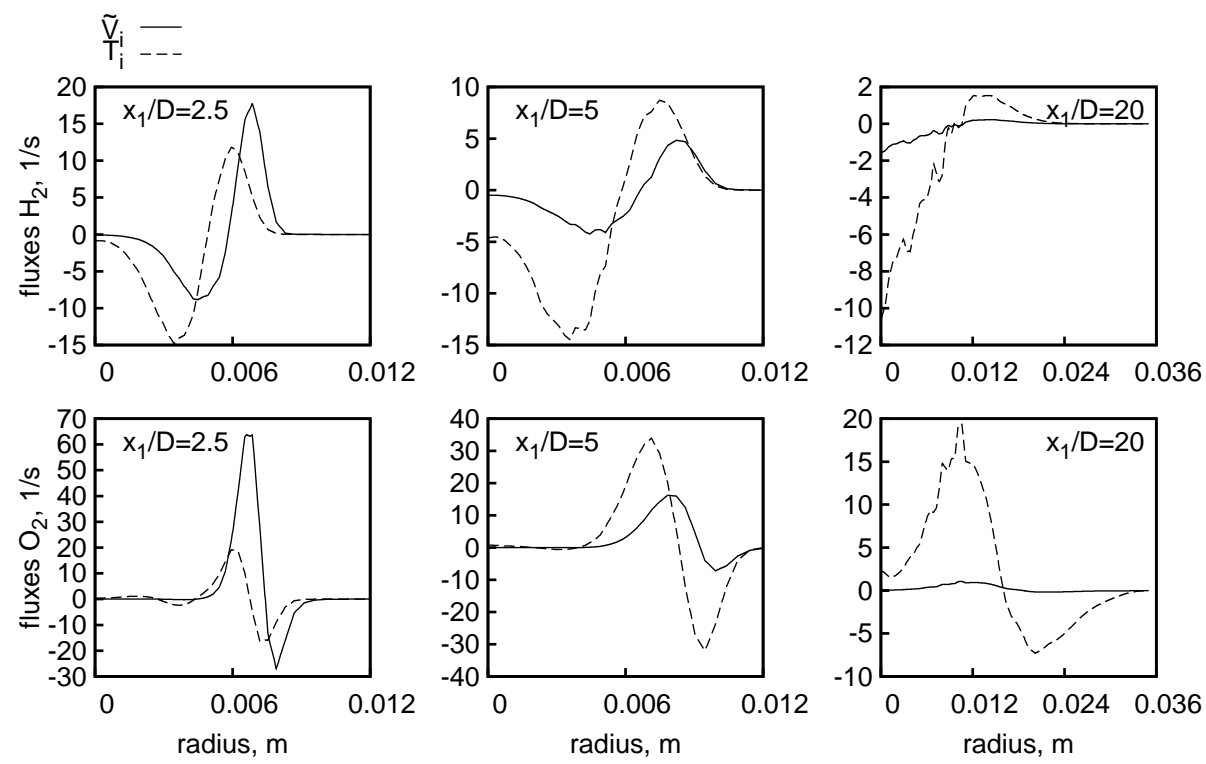

Figure 8: Magnitude of turbulent and molecular fluxes for $\tilde{Y}_{\mathrm{H}_{2}}$ and $\tilde{Y}_{\mathrm{O}_{2}}$.

turbulence Reynolds numbers are limited to the range of the pipe radius, i.e. $4 \mathrm{~mm}$. This indicates that the largest turbulent eddies appear in the fuel jet. Outside the fuel jet $R e_{L}$ drops to zero rapidly. Thus, no large scale turbulent motions exist outside the jet to enhance the mixing. Therefore, it is expected that molecular and turbulent transport are at least of the same order of magnitude. The situation is quite similar at $x_{1} / \mathrm{D}=5$. Further downstream at $x_{1} / \mathrm{D}=20$, the turbulence Reynolds number increases significantly to maximum values of $R e_{L} \approx 1200$. These high values are preserved over the entire span of the flame. Turbulent mixing should be dominant at this position.

To study quantitatively the importance of modeled molecular and turbulent transport, the simplified MDF transport equation is considered (Einstein 
notation)

$$
\begin{aligned}
\frac{\partial \mathscr{F}_{\phi}}{\partial t} & -\frac{\partial}{\partial x_{\alpha}}\left(\langle\rho\rangle D_{T} \frac{\partial}{\partial x_{\alpha}}\left(\frac{\mathscr{F}_{\phi}}{\langle\rho\rangle}\right)\right)+\frac{\partial}{\partial h}\left(\left(\tilde{V}_{h}-\frac{C_{\phi}}{2} \omega(h-\tilde{h})\right) \mathscr{F}_{\phi}\right) \\
& +\frac{\partial}{\partial Y_{i}}\left(\left(\tilde{V}_{Y i}-\frac{C_{\phi}}{2} \omega\left(Y_{i}-\tilde{Y}_{i}\right)\right) \mathscr{F}_{\phi}\right)=0,
\end{aligned}
$$

i.e. the convective transport and the chemical source terms are neglected in Eq. (14). Based on this MDF transport equation, the transport equations for the Favre averaged mass fractions of the educts, $\tilde{Y}_{\mathrm{O}_{2}}$ and $\tilde{Y}_{\mathrm{H}_{2}}$, read

$$
\begin{aligned}
& \frac{\partial \tilde{Y}_{\mathrm{O}_{2}}}{\partial t}=\mathcal{T}_{\mathrm{O}_{2}}+\tilde{V}_{Y \mathrm{O}_{2}}, \\
& \frac{\partial \tilde{Y}_{\mathrm{H}_{2}}}{\partial t}=\mathcal{T}_{\mathrm{H}_{2}}+\tilde{V}_{Y \mathrm{H}_{2}}
\end{aligned}
$$

The term $\mathcal{T}_{i}$ denotes the turbulent transport given by the gradient diffusion model (Einstein notation)

$$
\mathcal{T}_{i}=\frac{\partial}{\partial x_{\alpha}}\left(D_{T} \frac{\partial \tilde{Y}_{i}}{\partial x_{\alpha}}\right)
$$

whereas the Favre averaged molecular fluxes $\tilde{V}_{Y i}$ were introduced in section 2 . Both terms are evaluated in Fig. 8 for case 2 over the radius at the same positions as before. Since fuel is transported away from the jet and oxidizer towards it, the signs of the fluxes of $\mathrm{O}_{2}$ and $\mathrm{H}_{2}$ are reverse. Comparing the magnitudes of molecular and turbulent fluxes, i.e. of $\mathcal{T}_{i}$ and $V_{Y i}$, proves, that both terms are at least of the same order of magnitude close to the nozzle at $x_{1} / \mathrm{D}=2.5$ and $x_{1} / \mathrm{D}=5$. In order to compare the fluxes of $\mathrm{H}_{2}$ to the fluxes $\mathrm{O}_{2}$, the fluxes must be normalized first. It is obvious from the definition of the molecular flux $\tilde{V}_{Y i}$ in section 2 and the turbulent flux in Eq. (33), that the fluxes scale with the magnitude of the mass fraction gradient, which again 

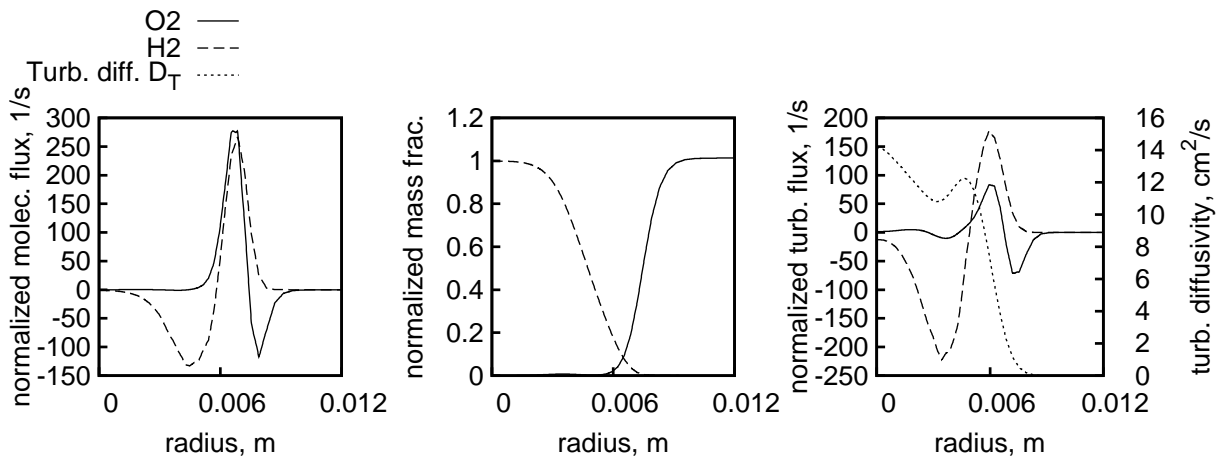

Figure 9: Normalized molecular fluxes, mass fractions and turbulent fluxes of $\mathrm{O}_{2}$ and $\mathrm{H}_{2}$ mass fraction at $x_{1} / \mathrm{D}=2.5$.

depends on the absolute magnitude of the species mass fraction. In order to eliminate this scaling effect, the flux of $\mathrm{O}_{2}$ is normalized by the maximum $\mathrm{O}_{2}$ mass fraction of 0.233 , whereas the $\mathrm{H}_{2}$ flux is normalized by the maximum $\mathrm{H}_{2}$ mass fraction of 0.06667. In this way the turbulent and molecular fluxes of different species can be compared with each other as illustrated in Fig. 9 at $x_{1} / \mathrm{D}=2.5$. It turns out, that in steady state the normalized molecular fluxes of $\mathrm{O}_{2}$ and $\mathrm{H}_{2}$ are of the same order of magnitude. This is surprising at first sight, since a larger flux $\mathrm{H}_{2}$ would be expected due to its higher diffusivity. This contradiction is clarified by considering the radial profiles of the normalized $\mathrm{H}_{2}$ and $\mathrm{O}_{2}$ mass fractions in Fig. 9. Since the highly diffusive $\mathrm{H}_{2}$ penetrates further into the surrounding air than $\mathrm{O}_{2}$ is capable to penetrate towards the jet centerline, the steady state profile of normalized $\mathrm{H}_{2}$ mass fraction is wider compared to the profile of normalized $\mathrm{O}_{2}$ mass fraction. Therefore, the spatial gradient of $\mathrm{H}_{2}$ mass fraction is smaller than the spatial gradient of $\mathrm{O}_{2}$ mass fraction. The smaller gradient of $\mathrm{H}_{2}$ in Fick's law is then balanced by the larger diffusion coefficient of $\mathrm{H}_{2}$ resulting thus 
in a normalized molecular flux which has the same order of magnitude as the normalized flux of $\mathrm{O}_{2}$. In the light of this discussion it is surprising that the normalized turbulent flux of $\mathrm{O}_{2}$ is smaller than the normalized turbulent flux of $\mathrm{H}_{2}$. This again is an outcome of the spatial variation of the turbulent diffusivity, which is needed to evaluate the turbulent flux in Eq. (33). The turbulent diffusivity is included in the plot of the normalized turbulent fluxes in Fig. 9. It is obvious, that there is a strong spatial variation of the turbulent diffusivity. The maxima are found in the center of the turbulent fuel jet and within the shear layer. Towards the surrounding air the turbulent diffusivity drops rapidly. Thus, the normalized turbulent flux of $\mathrm{O}_{2}$ is smaller than the normalized flux of $\mathrm{H}_{2}$ due to the larger turbulent diffusivity in the fuel jet. In Fig. 8, therfore, the molecular transport for the species $\mathrm{O}_{2}$ is significantly larger than the turbulent transport at $x_{1} / \mathrm{D}=2.5$. Thus, close to the nozzle molecular transport is important and must be included in the model. Further away from the nozzle at $x_{1} / \mathrm{D}=20$ the molecular fluxes almost vanish and turbulent fluxes dominate the mixing as shown in Fig. 8.

One disadvantage of studying Eqs. (31) and (32) is that the contribution of the IEM model to the mixing does not appear. Therfore, the mixedness of the educts is studied next, which we introduce in analogy to the definition given by Cetegen and Aguirre [46] as the Favre averaged product of educt mass fractions, i.e. $\widehat{Y_{\mathrm{O}_{2}} Y_{\mathrm{H}_{2}}}$. This parameter is zero in the fuel $\left(Y_{\mathrm{O}_{2}}=0\right.$ and $\left.Y_{\mathrm{H}_{2}}=0.06667\right)$ and oxidizer $\left(Y_{\mathrm{H}_{2}}=0\right.$ and $\left.Y_{\mathrm{O}_{2}}=0.233\right)$ stream because no mixture of educts is present. For intermittent, fully turbulent flows $\widetilde{Y_{\mathrm{O}_{2}} Y_{\mathrm{H}_{2}}}$ remains zero, since the concentration fields remain segragated (note, however, 
that the Favre averages $\tilde{Y}_{\mathrm{H}_{2}}$ and $\tilde{Y}_{\mathrm{O}_{2}}$ are non-zero for intermittency). If mixing is successful, the parameter becomes positive. Thus, $\widetilde{Y_{\mathrm{O}_{2}} Y_{\mathrm{H}_{2}}}$ is also a qualitative measure for the "reactiveness" of a mixture. Using Eq. (30) the transport equation

$$
\frac{\partial \widehat{Y_{\mathrm{O}_{2}} Y_{\mathrm{H}_{2}}}}{\partial t}=\mathcal{T}_{\mathrm{O}_{2} \mathrm{H}_{2}}-\mathcal{D}_{\mathrm{O}_{2} \mathrm{H}_{2}}+\mathcal{M}_{\mathrm{O}_{2} \mathrm{H}_{2}}
$$

is obtained, where the term (Einstein notation)

$$
\mathcal{T}_{\mathrm{O}_{2} \mathrm{H}_{2}}=\frac{\partial}{\partial x_{\alpha}}\left(D_{T} \frac{\partial \widehat{Y_{\mathrm{O}_{2}} Y_{\mathrm{H}_{2}}}}{\partial x_{\alpha}}\right)
$$

stems from the gradient diffusion model in Eq. (2) and represents the spatial transport due to turbulent velocity fluctuations. The terms $\mathcal{D}_{\mathrm{O}_{2} \mathrm{H}_{2}}$ and $\mathcal{M}_{\mathrm{O}_{2} \mathrm{H}_{2}}$ are artefacts of the diffusive flux decomposition in Eqs. (5) and (6). The term

$$
\mathcal{D}_{\mathrm{O}_{2} \mathrm{H}_{2}}=C_{\phi} \omega \widehat{Y_{\mathrm{O}_{2}}^{\prime \prime} Y_{\mathrm{H}_{2}}^{\prime \prime}}
$$

stems from the IEM closure for fluctuating conditional diffusive fluxes, whereas

$$
\mathcal{M}_{\mathrm{O}_{2} \mathrm{H}_{2}}=\tilde{V}_{Y_{\mathrm{O}_{2}}} \tilde{Y}_{\mathrm{H}_{2}}+\tilde{V}_{\mathrm{Y}_{2}} \tilde{Y}_{\mathrm{O}_{2}}
$$

includes the contribution of the Favre averaged diffusive fluxes. The three terms, i.e. $\mathcal{T}_{\mathrm{O}_{2} \mathrm{H}_{2}},-\mathcal{D}_{\mathrm{O}_{2} \mathrm{H}_{2}}$, and $\mathcal{M}_{\mathrm{O}_{2} \mathrm{H}_{2}}$ are evaluated in Fig. 10 at the same locations as the fluxes in Fig. 8. Case 2 is chosen here again since it employs the largest number of particles per cell, thus enabling an evaluation of $\widehat{Y_{\mathrm{O}_{2}} Y_{\mathrm{H}_{2}}}$ and $\widehat{Y_{\mathrm{O}_{2}}^{\prime \prime} Y_{\mathrm{H}_{2}}^{\prime \prime}}$ required in Eqs. (35) and (36) respectively with a small stochastic noise. The molecular flux $\mathcal{M}_{\mathrm{O}_{2} \mathrm{H}_{2}}$ (stemming from the average diffusion) dominates the mixing rate at $x_{1} / \mathrm{D}=2.5$. Compared to this term, 

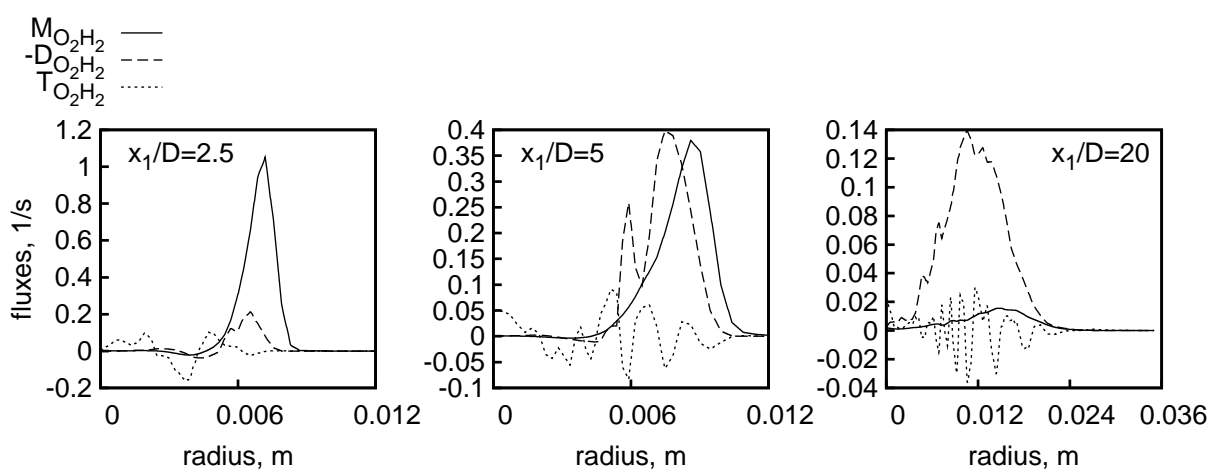

Figure 10: Magnitude of turbulent and molecular fluxes for $\widetilde{\mathrm{Y}_{\mathrm{O}_{2}} Y_{\mathrm{H}_{2}}}$.

the contribution of the IEM model to the mixing rate is one order of magnitude smaller. Also the contribution of the turbulent transport is negligible at this position. This explains why it is necessary to include molecular diffusion in order to stabilize the flame: The IEM model contribution is too small to obtain a reactive mixture close to the flame root. Further away from the nozzle at $x_{1} / \mathrm{D}=5$ the contribution of the diffusive fluxes decreases and the terms $-\mathcal{D}_{\mathrm{O}_{2} \mathrm{H}_{2}}$ and $\mathcal{M}_{\mathrm{O}_{2} \mathrm{H}_{2}}$ are of the same order of magnitude. Finally at $x_{1} / \mathrm{D}=20$ the term $\mathcal{M}_{\mathrm{O}_{2} \mathrm{H}_{2}}$ almost vanishes and the IEM model clearly dominates. The contribution of turbulent transport $\mathcal{T}_{\mathrm{O}_{2} \mathrm{H}_{2}}$ to the overall mixing rate seems to be small throughout the whole flame. Note, however, that the evaluation of this term is difficult, since no time averaged data of the variable $\widehat{Y_{\mathrm{O}_{2}} Y_{\mathrm{H}_{2}}}$ are available in order to evaluate the gradient of $\widetilde{Y_{\mathrm{O}_{2}} Y_{\mathrm{H}_{2}}}$. Latter quantity is required in Eq. (35) for the evaluation of $\mathcal{T}_{\mathrm{O}_{2} \mathrm{H}_{2}}$. The same applies to $\widehat{Y_{\mathrm{O}_{2}}^{\prime \prime} Y_{\mathrm{H}_{2}}^{\prime \prime}}$ where time averaged data are not available either. The lack of time averaged data explains the stochastic noise observed in Fig. 10. 


\subsection{Differential diffusion effects}

In subsection 5.3 the importance of molecular transport at the flame root is demonstrated. The capability of the presented model to predict differential diffusion is analyzed next. Meier et al. [22] report superequilibrium temperatures in the near field of the flame, which are caused by differential diffusion. In order to investigate this effect, temperature is plotted as a function of oxygen elemental mixture fraction at different axial positions downstream the nozzle. The oxygen elemental mixture fraction $Z_{O}$ is defined by

$$
Z_{O}=\frac{\mathcal{Z}_{O}-\mathcal{Z}_{O, O X}}{\mathcal{Z}_{O, F U}-\mathcal{Z}_{O, O X}}
$$

in terms of the oxygen elemental mass fraction $\mathcal{Z}_{O}$, where the indices $O X$ and $F U$ denote values of $\mathcal{Z}_{O}$ in the oxidizer and fuel stream respectively. As in the work of Meier et al. [22] scatterplots of temperature, which are obtained in the calculation from the data of the stochastic particles, are evaluated. Case 4 (see Tab. 1) is chosen since it has the highest mesh resolution in the near field. The results are shown in Fig. 11 together with the experimental data. To ease the orientation in the diagram, curves of the chemical equilibrium are included together with computations of a laminar counterflow diffusion flame at a strain rate of $181 / \mathrm{s}$. These computations are carried out with the software package Cantera [47]. The software package allows to include multi species diffusion for the laminar flame calculations. The effect of differential diffusion is clearly visible in the experimental data at $x_{1} / \mathrm{D}=2.5$. Here the equilibrium temperature is significantly lower than the flame temperatures observed in the experiments, which is caused by differential diffusion. The experimental data correlate well with the results of the transported PDF 


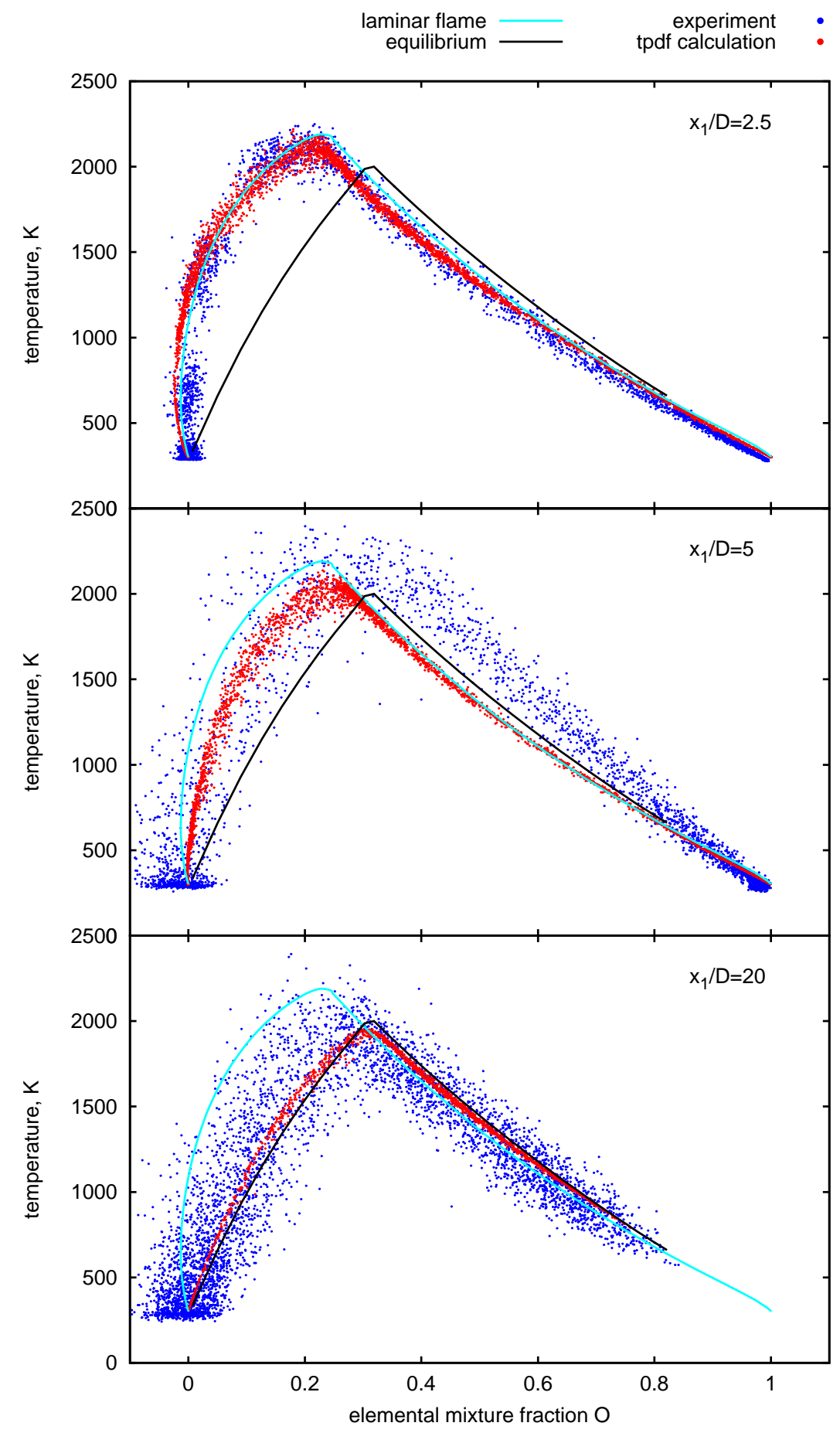

Figure 11: Temperature as function of oxygen elemental mixture fraction. 


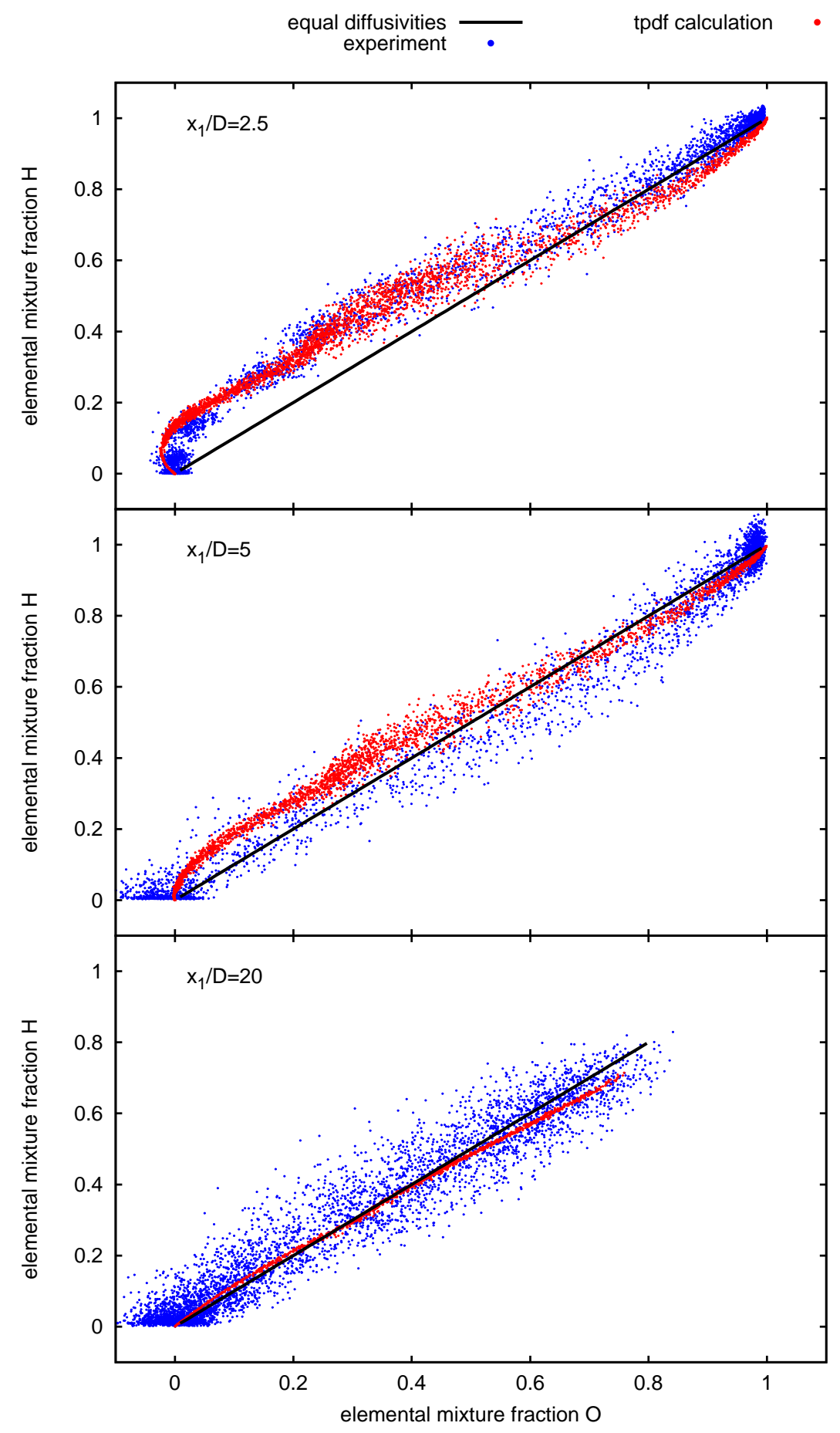

Figure 12: Correlation of elemental mixture fractions of $\mathrm{H}$ and $\mathrm{O}$. 
method and the laminar flame computation. Thus, in regions where turbulent mixing is negligible, the model is indeed capable to account for mixing due to differential diffusion. Further downstream at $x_{1} / \mathrm{D}=5$ the effect of differential diffusion is still visible but already weakened by turbulence. At $x_{1} / \mathrm{D}=20$ the experimental data and the transported PDF computation collapse on the equilibrium curve. Here turbulence governs the mixing. In comparison to the calculation, the scatter in the experiment is wider. This is due to experimental errors. For an oxygen elemental mixture fraction of zero for example, the scatter in the experiment must reduce to zero, since this state corresponds to pure air. There is, however, still a scatter in the experimental data visible and its magnitude at that point is a measure for the experimental uncertainties. Another way to analyze effects of differential diffusion in turbulent flames is to evaluate elemental mixture fractions as suggested by Barlow et al. [48]. Here the elemental mixture fraction of hydrogen

$$
Z_{H}=\frac{\mathcal{Z}_{H}-\mathcal{Z}_{H, O X}}{\mathcal{Z}_{H, F U}-\mathcal{Z}_{H, O X}}
$$

is introduced in analogy to Eq. (38). Since the mixture fractions are normalized element mass fractions, the transport equation for the elemental mixture fractions can be easily derived from the transport equation of element mass fraction given by Peters [40]. This yields (Einstein notation)

$$
\rho \frac{\partial Z_{i}}{\partial t}+\rho u_{\alpha} \frac{\partial Z_{i}}{\partial x_{\alpha}}=\frac{\partial}{\partial x_{\alpha}}\left(\rho D \frac{\partial Z_{i}}{\partial x_{\alpha}}\right), \quad i=\mathrm{O} ; \mathrm{H}
$$

if equal diffusivities $D$ are assumed. Thus, for equal diffusivities the elemental mixture fractions of hydrogen and oxygen obey the same transport equation. Since the boundary conditions for the solution of Eq. (40) are same for both 
elemental mixture fractions (i.e. $Z_{i}=0$ in the oxidizer stream and $Z_{i}=1$ in the fuel stream, zero flux across wall boundaries) the solutions of $Z_{H}$ and $Z_{O}$ are identical, i.e.

$$
Z_{H}\left(x_{\alpha}, t\right)=Z_{O}\left(x_{\alpha}, t\right)
$$

provided that both variables share the same initial conditions. For differential diffusion, however, this linear relation is not valid and deviations are expected. To verify this, the elemental mixture fraction of hydrogen $Z_{H}$ is plotted as a function of the oxygen elemental mixture fraction $Z_{O}$ in Fig. 12 . The black curve indicates the linear behavior expected for equal diffusivities. As in Fig. 11 both experimental and computational data of the transported PDF method are evaluated here. The agreement between computation and experiment is excellent: Close to the nozzle at $x_{1} / \mathrm{D}=2.5$ the experimental and numerical data clearly depart from this linear relation. Between $Z_{O}=0$ and $Z_{O}=0.6$ the elemental mixture fraction of hydrogen is significantly higher than in the case of equal diffusivities. This confirms that molecular diffusivities are not equal. Towards the fuel rich end, i.e. between $Z_{O}=0.6$ and $Z_{O}=1$, the effect is less pronounced. Further downstream at $x_{1} / \mathrm{D}=5$ the effect of differential diffusion is still visible between $Z_{O}=0$ and $Z_{O}=0.4$ where $Z_{H}$ lies above the line of equal diffusivities. At $x_{1} / \mathrm{D}=20$ turbulent mixing dominates the process and the elemental mixture fractions collapse on the line of equal diffusivities.

\section{Summary and conclusions}

In this paper a model approach similar to the method of McDermott et al. [18] is derived for hybrid RANS/transported PDF calculations to model molec- 
ular transport in transported PDF computations. It is successfully demonstrated with the example of heat transfer in channel and pipe flows that this model is capable to treat molecular transport of heat in wall bounded flows accurately. Models which employ the high Reynolds number assumption and which therefore neglect the contribution of Favre averaged molecular diffusive transport fail in the near wall region. Thus, this model approach is of paramount importance for the calculation of combustors, where wall heat losses must be included. The model is further applied to the computation of a turbulent, non-premixed hydrogen-air flame. Since this flame is stabilized by differential diffusion at its flame root, it is impossible to get a "burning solution" without modeling molecular diffusion. The accuracy of these computations is limited by the fidelity of the $k-\varepsilon$ turbulence model used. However the general flame structure can be reproduced well in the light of this limitation. Of particular interest is also the quantitative prediction of the model in the nozzle near field where molecular transport governs the mixing of reactants as demonstrated by an evaluation of the fluxes. Comparisons with experimental data show that the model can reproduce these effects of differential diffusion accurately. To our knowledge, the present paper is the first work, where the stabilization of a non-piloted, non-premixed, turbulent jet flame through differential diffusion at its laminar flame root is successfully modeled in hybrid RANS/transported PDF calculations. It is concluded, that neglecting molecular transport may yield in the worst case to global flame extinction as this work demonstrates. 


\section{Acknowledgments}

The authors thank the Jülich Supercomputing Centre for the computational time provided under the NIC project number 5904.

\section{References}

[1] R. Curl, A.I.Ch.E. Journal 9 (1963) 175-181.

[2] J. Villermaux, J. Devillon, in: Second International Symposium on Chemical Reaction Engineering, Elsevier, New York, 1972, pp. 1-13.

[3] C. Dopazo, E. O’Brien, Acta Astronautica 1 (1974) 1239-1266.

[4] J. Janicka, W. Kolbe, W. Kollman, Journal of Non-Equilibrium Thermodynamics 4 (1979) 47-66.

[5] S. Pope, Combustion Science and Technology 28 (1982) 131-135.

[6] P. Nooren, H. Wouters, T. Peeters, R. D., U. Maas, D. Schmidt, Combustion Theory and Modelling 1 (1997) 79-96.

[7] R. O. Fox, P. Yeung, Physics of Fluids 15 (2003) 961-985.

[8] D. Meyer, P. Jenny, Physics of Fluids 18 (2006) 035105-1 - 035105-15.

[9] D. W. Meyer, P. Jenny, Combustion and Flame 155 (2008) 490-508.

[10] D. W. Meyer, P. Jenny, in: Proceedings of the Combustion Institute, volume 32, pp. 1613-1620.

[11] D. W. Meyer, P. Jenny, Journal of Computational Physics 228 (2009) $1275-1293$. 
[12] S. Pope, Theoretical and Computational Fluid Dynamics 2 (1991) 255270.

[13] F. Gao, E. E. O’Brien, Physics of Fluids A 3 (1991) 956-959.

[14] H. Chen, S. Chen, R. H. Kraichnan, Physical Review Letters 63 (1989) 2657-2660.

[15] S. Subramaniam, S. Pope, Combustion and Flame 115 (1998) 487-514.

[16] R. O. Fox, Computational Models for Turbulent Reacting Flows, Cambridge University Press, 2003.

[17] J. Pozorski, J.-P. Minier, International Journal for Heat and Fluid Flow 27 (2006) 867-877.

[18] R. McDermott, S. B. Pope, Journal of Computational Physics 226 (2007) 947-993.

[19] S. Viswanathan, H. Wang, S. B. Pope, Journal of Computational Physics 230 (2011) 6916-6957.

[20] P. H. Abbrecht, S. W. Churchill, A.I.Ch.E. Journal 6 (1959) 268-273.

[21] D. Pfuderer, A. Neuber, G. Früchtel, E. Hassel, J. Janicka, Combustion and Flame 106 (1996) 301-317.

[22] W. Meier, S. Prucker, M.-H. Cao, W. Stricker, Combustion Science and Technology 118 (1996) 293-312.

[23] J. D. Anderson, Jr., Hypersonic and High Temperature Gas Dynamics, McGraw-Hill Book Company, 1989. 
[24] S. B. Pope, Progress in Energy and Combustion Science 11 (1985) 119 192.

[25] S. B. Pope, Combustion and Flame 27 (1976) 299-312.

[26] T. Coffee, J. Heimerl, Combustion and Flame 43 (1981) 273-289.

[27] P. Gerlinger, Numerische Verbrennungssimulation: Effiziente numerische Simulation turbulenter Verbrennung, Springer-Verlag Berlin Heidelberg, 2005.

[28] R. Courant, D. Hilbert, Methods of Mathematical Physics, Interscience Publishers, a division of John Wiley \& Sons, New York, London, 1962.

[29] A. Fiolitakis, P. R. Ess, P. Gerlinger, M. Aigner, in: Proceedings of the 50th AIAA Aerospace Sciences Meeting including the New Horizons Forum and Aerospace Exposition, AIAA 2012-0179.

[30] J. H. Ferziger, P. Milovan, Computational Methods for Fluid Dynamics, Springer-Verlag Berlin Heidelberg New York, 2002.

[31] H. Möbus, Euler- und Lagrange-Monte-Carlo-PDF-Simulation turbulenter Strömungs-, Mischungs- und Verbrennungsvorgänge, Ph.D. thesis, Universität Stuttgart, 2001.

[32] W. Jones, B. Launder, International Journal of Heat and Mass Transfer 15 (1972) 301-314.

[33] D. C. Wilcox, AIAA Journal 26 (1988) 1299-1310. 
[34] H. Wang, S. B. Pope, Combustion Theory and Modelling 12 (2008) $529-544$.

[35] M. Muradoglu, P. Jenny, S. B. Pope, D. A. Caughey, Journal of Computational Physics 154 (1999) 342-371.

[36] A. Malhotra, S. Kang, International Journal of Heat and Mass Transfer 27 (1984) 2158-2161.

[37] S. B. Pope, Turbulent Flows, Cambridge University Press, 2009.

[38] A. Neuber, G. Krieger, M. Tacke, E. Hassel, J. Janicka, in: 11. TECFLAM-Seminar: Verbrennungsdiagnostik und Prozeßkontrolle, Arbeitsgemeinschaft TECFLAM, 1995, pp. 83-99.

[39] T.-C. Cheng, G. Fruechtel, A. Neuber, F. Lipp, E. P. Hassel, J. Janicka, Forschung im Ingenieurwesen - Engineering Research 61 (1995) 165-171.

[40] N. Peters, Turbulent Combustion, Cambridge monographs on mechanics, 2006.

[41] N. Peters, B. Rogg, Reduced Kinetic Mechanisms for Applications in Combustion Systems, Springer, 1993.

[42] San diego mechanism, Online, accessed Sep. 25th, 2008. http:// maeweb.ucsd.edu/ combustion/Mechanisms, 2008.

[43] A. Fiolitakis, P. Gerlinger, B. Noll, M. Aigner, W. Krebs, B. Wegner, in: Proceedings of the 48th AIAA Aerospace Sciences Meeting including the New Horizons Forum and Aerospace Exposition, AIAA 2010-608. 
[44] B. Launder, B. Sharma, Letters of Heat and Mass Transfer 1 (1974) $131-138$.

[45] H. Versteeg, W. Malalasekera, An Introduction to computational fluid dynamics: The finite volume method, Pearson Prentice Halls, 1995.

[46] B. Cetegen, J. Aguirre, Physics of Fluids 2 (1990) 2211-2216.

[47] D. Goodwin, in: M. Allendorf, F. Maury, F. Teyssandier (Eds.), Chemical Vapor Deposition XVI and EUROCVD 14, ECS Proceedings, volume 2003-08, pp. 155-162.

[48] R. S. Barlow, J. H. Frank, A. N. Karpetis, J.-Y. Chen, Combustion and Flame 143 (2005) 433-449. 